\title{
An Overview of Demand Side Management Control Schemes for Buildings in Smart Grids
}

Kosek, Anna Magdalena; Costanzo, Giuseppe Tommaso; Bindner, Henrik W.; Gehrke, Oliver

Published in:

2013 IEEE International Conference on Smart Energy Grid Engineering (SEGE)

Link to article, DOI:

10.1109/SEGE.2013.6707934

Publication date:

2013

Link back to DTU Orbit

Citation (APA):

Kosek, A. M., Costanzo, G. T., Bindner, H. W., \& Gehrke, O. (2013). An Overview of Demand Side Management Control Schemes for Buildings in Smart Grids. In 2013 IEEE International Conference on Smart Energy Grid Engineering (SEGE) IEEE. https://doi.org/10.1109/SEGE.2013.6707934

\section{General rights}

Copyright and moral rights for the publications made accessible in the public portal are retained by the authors and/or other copyright owners and it is a condition of accessing publications that users recognise and abide by the legal requirements associated with these rights.

- Users may download and print one copy of any publication from the public portal for the purpose of private study or research.

- You may not further distribute the material or use it for any profit-making activity or commercial gain

- You may freely distribute the URL identifying the publication in the public portal 


\title{
An Overview of Demand Side Management Control Schemes for Buildings in Smart Grids
}

\author{
Anna Magdalena Kosek*, Giuseppe Tommaso Costanzo*, Henrik W. Bindner* and Oliver Gehrke* \\ *Technical University of Denmark, Department of Electrical Engineering \\ Energy System Operation and Management \\ Email: \{amko,guco,hwbi,olge\}@elektro.dtu.dk
}

\begin{abstract}
The increasing share of distributed energy resources and renewable energy in power systems results in a highly variable and less controllable energy production. Therefore, in order to ensure stability and to reduce the infrastructure and operation cost of the power grid, flexible and controllable demand is needed. The research area of demand side management is still very much in flux and several options are being presented which can all be used to manage loads in order to achieve a flexible and more responsive demand. These different control schemes are developed with different organization of the power sector in mind and thus can differ significantly in their architecture, their integration into the various markets, their integration into distribution network operation and several other aspects. This paper proposes a classification of load control policies for demand side management in smart buildings, based on external behavior: direct, indirect, transactional and autonomous control; internal operation: decision support system scope, control strategy, failure handling and architecture. This classification assists in providing an overview of the control schemes as well as different ways of representing a building.
\end{abstract}

\section{INTRODUCTION}

In most of today's power systems, nuclear, hydro and fossil power plants provide the majority of energy production [1], [2], while peak consumption is matched by regulation plants and power exchange between grids. However, in the last two decades factors such as the increased global energy demand, speculation on fossil fuels, and global warming have generated a high interest in Renewable Energy Resources (RES) and Electric Vehicles (EVs). For example, in Denmark the wind power is planned to supply approximately $50 \%$ of the demand by the year 2020 [3]. In this context, power systems will have to face challenges such as accommodating a highlyvariable and less controllable distributed energy production, and reducing the peak demand. In order to avoid or delay large investments in grid infrastructures and storage facilities, already existing consumption units can be enabled with control capabilities. Demand Side Management (DSM) is a promising technology for power balancing in future energy systems.

A classification of control policies for DSM focusing on smart buildings, presented in this paper, can help with the selection of a DSM controller for a specific application, provide comparison criteria between technologies, and help in the standardization process.

$\mathrm{WBCSD}^{2}$ estimates that in most countries buildings account approximately for the $30-40 \%$ of total energy consumption [4], therefore controlling buildings with Home Automa-

\footnotetext{
${ }^{2}$ The World Business Council for Sustainable Development
}

tion Systems (HAS) with remote control can enhance overall demand flexibility. It is assumed, in this context, that a smart building is equipped with controllable appliances and smart devices in order to enable flexible consumption. As many research projects have investigated different approaches to DSM in smart buildings, this paper presents an overview of selected DSM systems and proposes a classification for control schemes for Distributed Energy Resources (DERs).

This paper is organized as follows: in Section II control policies are classified depending on external behavior. This section presents general DSM control policies not only for buildings, but any DER present in the Distribution Grid. In Section III control policies are classified by internal operation of the HAS, and in Section IV building representation is described. Section V presents an overview of selected DSM and HAS systems and describes how the proposed classification can be applied to some of the related control policies. DER Control comparison criteria and useful simulation tool are introduced in section VI. Conclusions and future work are outlined in Section VII.

\section{EXTERNAL BEHAVIOR}

Independently of the reference architecture chosen for the future Smart Grid, different types of interactions between actors can be proposed and DER control policies defined. The external behavior characterizes the interactions of DER with other entities in the architecture, such as other controllers, utilities or energy traders in terms of communication type and information exchange.

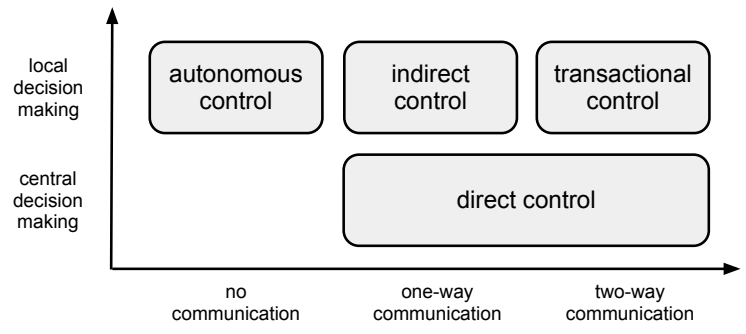

Fig. 1. DER control matrix for demand side management.

The classification based on external behavior contains possible mechanisms within DER of reacting on signals received form other actors in the Smart Grid. In the Demand Side Management domain control policies are usually divided into direct and indirect, for example as proposed in [5] and [6]. In this paper a DSM control policies classification is presented 
proposing four categories: autonomous, direct, indirect and transactional control. Figure 1 depicts a proposed classification mapped onto communication type and the location of the decision making process. Direct control uses an external controller with a central decision making process to determine DER operation, where in case of autonomous, indirect and transaction control policies the DER is responsible for local decisions.

\section{A. Direct Control}

Direct control is a technique based on issuing specific commands to controllable DERs. The decision on DER operation is taken by an external controller that embeds the main intelligence and has a knowledge of the DER status. In the direct control scheme an external controller has specific and detailed information about the DER operation.

A direct controller uses two-way or one-way unicast communication to exchange information with a DER. In case of one-way communication a DER is obliged to follow control commands. The only deviation from expected behavior can occur due to operational limit violation or failures. In case of bidirectional communication each DER is obliged to acknowledge the received command. A DER informs the external controller whether the control command can be executed or not, and provides the controller with the DER status. Commands sent by the external controller may vary depending on the DER type and communication protocol.

Gehrke et. al [7] presented direct load control schemes, depending on the information type exchanged between the controller and a DER: deferred consumption, delta consumption, scheduled operation and direct power control. Load control schemes can be extended to cover both consumption and production, therefore DER operation control schemes are defined as follows:

1) Deferred operation: The consumption or production of a certain amount of energy is shifted in time, as presented in Figure 2(A). Neither the amount of consumed or produced power, nor duration of the operation is modified. The signal that is received by the DER is of type $\Delta t$, which represents a required delay of operation.

2) Delta operation: The amount of energy consumed or produced by the controlled DER is decreased or increased by an offset of $\Delta P$, called power difference, see Figure 2(B). Decrease in consumption might result in increase in duration of the operation, for example in operation of thermal loads like a heat-pump.

3) Scheduled operation: The DER is provided with an operation schedule $s$, consisting of time series of power set points and time stamps, where $s=\left\{\left(t_{i}, P_{i}\right)\right\}, i \in \mathbb{N}$, as shown in Figure 2(C).

4) Direct power control: At run-time the DER is provided with a power set point, $P$, as depicted in in Figure 2(D).

Each DER is equipped with a local controller for lowlevel hardware control, which is responsible for performing the necessary actions in order to meet the requirements set by the external controller. A direct controller, by using an optimized mix of the direct control schemes presented above,
(A)

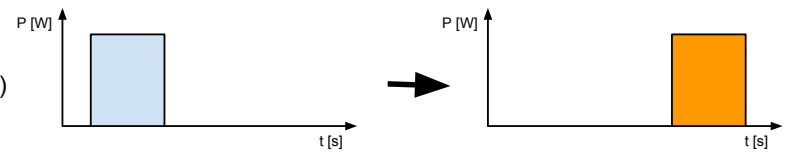

(B)

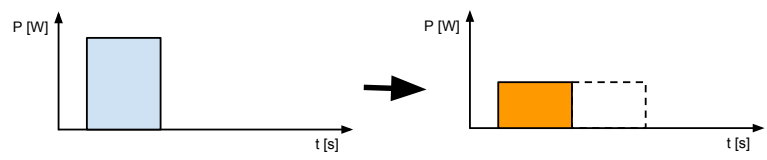

(C)
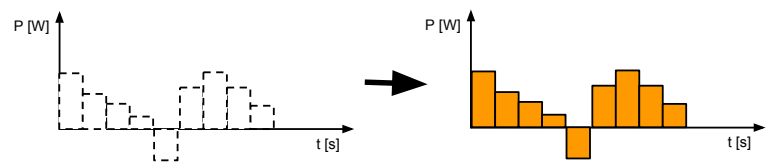

(D)

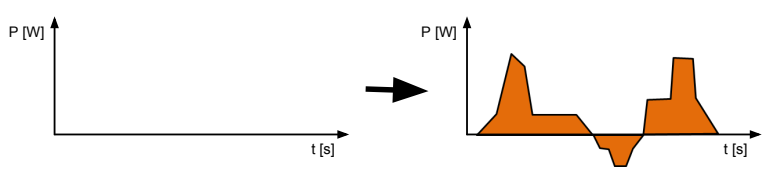

Fig. 2. Operation schemes for DER direct control: (A) Deferred operation, (B) Delta operation, (C) Scheduled operation, (D) Direct power control.

can operate units in order to provide such services as: voltage and frequency regulation, load shedding, valley filling. In the control-by-services scheme high level commands are issued to a DER to control a previously defined service. In this type of direct control a DER service, for example a voltage control, is decoupled from a DER type, for example a wind turbine [8].

\section{B. Indirect Control}

In a power system context, indirect control is a way of managing a DER by issuing signals which might or might not affect its operation. Heussen et. al. in [6] define two characteristics of the indirect control: "indirectness of the relationship between control objective and observables", and "local and independent decision making of the DER makes its behavior non-deterministic". The DER accepts the control signals but it is not obliged to either react to the signal or send any feedback. Lack of the feedback implies that indirect control requires only one-way communication to transfer the control signal from external controller to a DER. Indirect control can uses broadcast to propagate messages, any DER can simply choose to listen to commands at any time.

Scalability, as defined by Tanenbaum [9], is a characteristic of a system that remains effective when there is a significant increase in number of resources or users. Broadcast signals propagated in the indirect control scheme can be received by any number of units, in contrast to single addressing in unicast communication in the direct control scheme. Scalability of the indirect control makes it suitable for systems with high uncertainty in DER number, type and external influence factors, for example power systems with changing topology.

The indirect control advantages are simplified protocols, exchanged data and interfaces. DERs can follow thair private and dynamic objectives and prioritize local goals. In comparison to direct control, predictability of the DER response is lower, due to its non-deterministic behavior. To reduce mismatch between control objective and the actual response, DER models including relationship between signal and the response are needed [6]. If the field measurements of the power 
consumption are available, it is possible to infer reaction of a DER by the impact they have on the grid.

Heussen et.al. [6] classifie indirect control into four categories: control with indirect functional variables, independent local control, indirect control via price signals and control with internal market platform. In this paper independent local control (called here autonomous control) and control with internal market platform (called here transactional control) are separated from indirect control as depicted in Figure 1 and described in section II-D. Indirect control, following [6], is divided into two categories:

1) Control with indirect functional variables: A control scheme in which a signal containing a functional variable is sent from an external controller to a DER in order to influence the DER operation. An example of a functional variable can be a local set-point thermostat temperature in a heating system. The external controller objective is to modify heating system power consumption without knowledge of heaters state. Since the state is unknown and there is no control feedback, the system response is non-deterministic. Observable or estimated aggregated response, for example the heating system power consumption, can be used to generate the functional variable signal. Statistical models can be used to reduce the mismatch between the control objective and the system response.

2) Indirect control via price signals: A control scheme based on issuing energy prices to DERs in order to alternate its operation. Indirect control via price signals is further divided into instantaneous price operation and scheduled price operation. In the instantaneous price operation scheme the control signal consists of a single price $p$ and time stamp signal that states the time when the price becomes valid. In the scheduled price operation, the signal is a schedule $s$ of future prices, consisting of time series of prices and time stamps, where $s=\left\{\left(t_{i}, p_{i}\right)\right\}, i \in \mathbb{N}$.

\section{Transactional control}

Transactional control, called also market-based control [10], or control with internal market platform [6] refers to a control strategy based on negotiations in a bid-based market. In transactional control DERs are competing for one or more resources on an equilibrium market with the use of bids. After the end of the the transaction DERs can optimize their production or consumption with use of the equilibrium value determined by the market. The main goal of the transactional control is to distribute resources efficiently by taking into account correlated needs of different DERs [11]. A DER independently decides about the bidding amount and autonomously plan the control accordingly to the winning bid.

Price-based transactional control combines the price negotiation features with local biding strategy, resulting in resource allocation based on individual DER needs without revealing its operation strategy. The decisions about DER operation and offered bids are taken locally, the market is constructed to find a price equilibrium for participating DERs.

\section{Autonomous control}

DER controllers can be designed to take decisions based on local signals, such as frequency or voltage measurements.
In this way each single unit can participate in frequency or voltage regulation independently. Due to the use of local measurements, this kind of control is characterized by a fast response and it does not need a communication infrastructure or higher-level control entities. While such DER does not need any supervision, and works autonomously, it can provide a valuable local power system service.

In this section, the external behavior of the controller was characterized by direct, indirect, transactional and autonomous control mechanisms. Another characteristic on which it is possible to classify control policies is the internal DER operation, which is described in the following section. This classification focuses on a particular type of DER, a controllable building. In the context of the power system a smart building is referred to a controllable building capable of accepting commands from external controllers, managing and supervising the operation of all internal subsystems.

\section{INTERNAL OPERATION}

The internal operation of a controller in a building regards all the inner actions taken by the controller and it can be characterized by the scope of the decision support system, control strategy, failure handling and architecture.

\section{A. Decision support system scope}

Depending on the information a controller is provided with, it can operate on the basis of different techniques. In the domain of Demand Side Management three types of decision support system can be recognized based on the controller decision scope: past, present and future (Fig. 3).

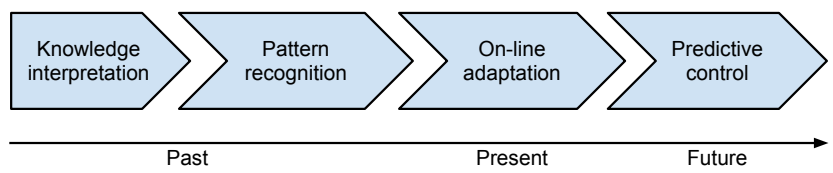

Fig. 3. Decision support system scopes.

1) Knowledge interpretation: The general knowledge about a specific domain is interpreted to support decision making mechanism. Knowledge can be fixed or learned form the historical data.

2) Pattern recognition: A decision is based on historical data by performing pattern recognition. Historical data may be both gathered at runtime, during device operation, or provided as a calibration set by the manufacturer. Historical data can also be used to track users behavior patterns, devices consumption models or to relate power consumption to time of day or day of week.

3) Online adaptation: Online adaptation is based on logic that uses information gathered at run-time to adapt to changing conditions and does not take into account the control actions taken previously.

4) Predictive control: A prediction of users behavior or devices power consumption is used for optimal scheduling of house power consumption. This prediction can be based on available models, historical data or both. Information provided 
by other entities, such as weather forecast source, can be used to plan unit operation to fit changing environmental conditions.

Intelligent controllers can combine presented scopes and use historical, live data and forecasts in their decision support system. For example a DER consumption model can be created from historical data and general domain knowledge and updated with live data. This model can be then used together with weather forecasts to produce a DER consumption prediction.

\section{B. Control strategy}

In the run-time a controller may be flexible toward a change of its control strategy. The control strategy can be classified as static, flexible and re-configurable. Static controllers work with a defined control strategy that is not changed, while flexible controllers implement several control strategies that can be switched during the unit operation on the basis of local measurements or third party information provided by external controllers. On the other hand, reconfigurable controllers are designed to modify the internal logic based on external influences. Advanced reconfigurable controllers can implement machine learning, defined as ability of computer systems to automatically improve with experience [12]. Although the controllers belonging to this latter class offer great flexibility, their behavior is subject to a continuous tuning process, therefore it might be difficult to predict.

\section{Failure handling}

Resilience, defined by Rieger [13], is an ability of a control system to remain effective despite of disturbances, assuring safe and secure operation of the controlled components. In the context of the power system, the ability to sustain grid services is connected to failure handling: detection, diagnosis, masking and tolerating. Faults can include hardware and software faults, for example fail-stop, crash or omission (including connection failures). In this section control schemes for failure handling are divided into two categories: mask or tolerate.

1) Mask failures: Failures that are detected can be hidden or made less critical. Software controllers using techniques to mask failures can try to reconnect to other software components, for example a database, switch to another source of data, for example search for the nearest weather station.

2) Tolerate failures: A controller implements failure toleration if it continues to work even if part of the external system or internal modules have failed. Some of the software modules of the controller can fail: communication interface, data logger, external data might not arrive when expected or databases can refuse the connection. A controller that tolerates failures can perform gentle degradation. In the case of severe faults in the external system, controller can switch to a simple autonomous control or apply default settings.

In the context of power system, the external controllability of a DER that implements failure masking or tolerance depends on the type of failure handling that was chosen for the controller architecture. DERs, whose operation is based on continuous information retrieval, such as price signals in indirect control or power set points in direct control scheme, get affected in their operation in case of failure of connection. In this case, the DER controller may switch to other optimization objectives, like maximization of user comfort or following a flexible behavior pattern. Therefore the DER is no longer offering services to the grid. A control policy can also be unaffected by faults when a DER performs a service that is independent of continuous communication with external controllers. For example, direct control with a schedule allows DERs to operate, once received the operation schedule, until the scheduled instructions finish without receiving any further information. Also DERs controlled with local signals can continue operating, since local measurements are independent of the communication with an external controller.

\section{Architecture}

The internal architecture of a controller managing a DER can be centralized, decentralized, distributed or hierarchical.

1) Centralized architecture: In the case of a centralized architecture, the central component has a complete view of the system and takes decisions regarding units operation. All decisions need to be made in the central component. Centralized software architecture brings advantages of concentrating the controller's functions in a single component, that is able to perform system level optimization, it is also able to judge system state and harmonize all performed actions. On the other hand the central component is both a single point of failure and might introduce the bottleneck of the system, therefore centralized architecture should be designed carefully.

2) Decentralized architecture: In the decentralized architecture, a control task is taken away from the central controller, decomposed into sub-tasks and delegated to different subsystems [14]. In the decentralized architecture there are no couplings between subsystems, their operation is autonomous.

3) Distributed architecture: In the case of distributed software architecture, subsystems take local decisions based on information exchange with other subsystems [14]. In this architecture subsystems might have the same or different and possibly conflicting goals [15]. Consensus can be reached by a negotiation process. Such a scheme allows cooperation, resources negotiation and exchange of information.

4) Hierarchical architecture: A system has a hierarchical architecture if different layers of abstraction can be distinguished [14]. Levels are introduced to reduce complexity, usually highest layer models system characteristics and lower layers represent detailed characteristics.

Multi-Agent Systems (MAS) is a very popular programming paradigm that allows implementation of all presented architectures. A MAS mostly implements distributed and hierarchical architectures, where responsive agents encapsulate different behavior and follow own goals while cooperating or competing with other agents for resources.

Optimal, reliable and flexible solutions can be achieved using any controller software architecture, and the choice depends on the application and system requirements. As it has been presented in this section, control policies can be classified by their internal operation. Characterization based on decision support system scope, control strategy, failure handling and software architecture were presented.

The concept of demand response uses the controllability of DERs to provide power system services. In order to control 
a building, a representation of the building and all devices consuming and producing power in it needs to be defined. Section IV presents a proposed building representation and its impact on the external building behavior towards other actors in the power system in direct, indirect and transactional control policies.

\section{BUILDING REPRESENTATION}

A building may not be viewed a uniform DER, it can a collection of separate subsystems, for example: HVAC, lighting systems, appliances and different devices coexisting in a space, all supplied from the same grid connection. How a single house is represented in the power system context is important for the control strategy. A building can be defined as a single DER or as a collection of systems that are defined as separate DERs as presented in Figure 4.

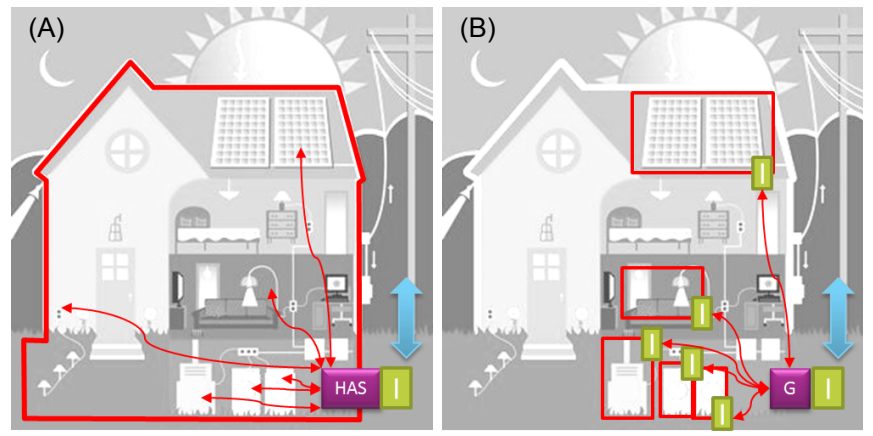

Fig. 4. Building representation the power system for control and aggregation. (A) building represented as a single DER, (B)- building represented as a collection of DERs, HAS - Home Automation System, G - gateway, Iinterface.

1) Building represented as a single DER: A building is a consumer or a prosumer (both consumer and producer) that can be controlled externally through an interface to an internal HAS. The HAS communicates with the external controller and operate devices in the house, as depicted in Figure 4(A). For the external controller a house is a controllable single unit. An interface to communicate between external controller and HAS need to be implemented.

2) Building represented as a collection of DERs: The building subsystems are for example heating, ventilation, hot water, lighting systems, appliances and all devices that are permanently or temporarily in the house. This list could also include electric vehicles or any other batteries, production units like: microCHP, generators, photovoltaics or wind turbines. In this case the gateway only passes the request from a high level controller to a DER and all subsystems are controlled individually. In this type of the building representation, a single HAS is replaced with a gateway translating from external to internal communication, as presented in Figure 4(B). In this case DERs need to implement an interface and data exchange protocols in order to be controlled remotely. These interfaces and protocols can differ form one DER to another, because every subsystem in the building can be controlled using a different technology implemented by different vendors.

Depending on which of the two building representations is used the control strategy differs. Following sections present influence of the building representation on direct, indirect and transactional control.

\section{A. Direct control}

If a building is represented as a single DER, it can be operated directly by an external controller. The HAS is responsible for dispatching tasks to different units and managing the requested power system service. In this case the existing HAS communication can be used to control devices. The HAS needs to implement an interface to the external controller and new power management functions responsible for providing power system services. Depending on the type of direct control, a HAS needs to implement additional features. For all direct control schemes a device operation schedule is needed. Since only the aggregated operation of the house is visible to the external controller, a HAS needs to create and update a schedule of operation. In order to properly schedule devices operation, especially in residential buildings, a HAS needs to recognize user preferences and behavior, and implement device models. Delta operation and direct power control schemes need on-line adaptation, since requests that are received concern only instantaneous consumption. Control signals received in deferred and scheduled operation schemes contains information about the future operation, therefore optimal device operation schedule can be produced.

In case of representing a house as a collection of DERs, all subsystems need to be individually controlled. Since many technologies can co-exist in one building, either standardized interfaces to DER should be used or the external controller needs to implement many specific interfaces to DERs. Since there is no HAS controlling the total house consumption and production, DER aggregated operation needs to be managed at the external controller level.

\section{B. Indirect control}

For a building represented as a single DER, a price signal can be sent to a HAS in order to control it indirectly. In the case of the instantaneous price operation scheme, a single price signal is used to influence the building operation, therefore HAS needs on-line adaptation and a mechanism to recognize if the price is low or high, usually based on historical data and user preferences. In the case of the scheduled price operation scheme, the HAS uses a prices signal to schedule devices' operation or plan the future operation of the entire building. In this case HAS internal communication infrastructure can be reused, because devices does need to receive price signals, but receive only control signals.

If a building is represented as a collection of DERs, the price signal is passed to individual DERs present in the house. The control decision is taken locally in the DER based on the received price. The DER need to interpret the price and, depending on the implemented indirect control scheme, adapt online to the received price or use prediction and received future energy prices to schedule its own operation. The existing HAS communication in the building can be reused, but the communication protocols and DER interface needs to be implemented to be able to pass the price signal to a DER.

\section{Transactional control}

With transactional control, a building that is represented asa single DER acts as a single entity bidding in a local market. The HAS coordinates and schedules operation of the 
entire building depending on the winning bid. If a building is represented as a collection of DERs, every subsystem can bid directly on the local market. Additional coordination between subsystems connected to a single point in the power grid might be needed to execute committed bids.

As discussed in this section, the representation of a building has an influence on the DER control scheme. For a building represented as a collection of DERs more coordination is required on the level of the external controller, as separate subsystems are connected to a single point in the power grid.

\section{OVERVIEW OF SELECTED DSM SYSTEMS AND HAS}

In this section examples of proposed controllers and architectures in the smart grids research domain are presented. The section is organised according to the presented control classification, dividing the existing research into external behaviour and internal operation, focusing on the home automation systems. Examples of different representation if the building are included in the presented overview.

\section{A. External behaviour}

1) Direct control: A Virtual Power Plant (VPP) is a flexible representation of a group of responsive DERs [16]. A VPP is created to represent it in a single portfolio of services to the power system. An example of a VPP using direct control is Power Hub [17], [18], a research project developed and implemented by Dong Energy ${ }^{1}$, a Danish energy utility, power producer and energy trader. PowerHub is a VPP directly controlling consumption and production units, using load and flexibility prediction tools, and providing an interface to use the VPP in connection to commercial ancillary services and energy markets. Power Hub's external controller uses two-way communication to retrieve DER's 24 hour operation schedule with operational flexibility, which defines acceptable variations from the given schedule. The Power Hub external controller manages DERs directly by sending start/stop commands and continuous varying consumption set point to the DER (power in $\mathrm{kW}$ ).

2) Indirect control: A VPP can also use the indirect control scheme to manage DERs. Price-responsive controllers for power systems were proposed in [19], and have been a subject of different research and demonstration projects [20], [21], [22]. An extended overview of indirect control challenges and technical considerations were presented in [23]. Examples of indirect control schemes presented in this paper can be found in [6]. Price signals propagated in the indirect control scheme need to be adapted to the aggregated response received form the VPP, price signal evaluation with use of models, forecast and hardware-in-the-loop method was presented in [24].

3) Transactional control: PowerMatcher [25] is an example of a demand response architecture using price-based transactional control scheme. PowerMatcher is based on a multi-agent platform for the integration and management of small and medium-sized DERs in the low voltage grid [25]. In the PowerMatcher platform, the distribution grid is clustered into logical trees, which have a single root, called Auctioneer agent. A cluster of DERs is managed by the Auctioneer to

\footnotetext{
${ }^{1}$ www.dongenergy.dk
}

fulfil a specific goal, for example providing a power system service as a VPP. The Auctioneer operates a local energy poolmarket within the cluster, on which the DERs can place bids. After all bids are collected, the Auctioneer agent searches the equilibrium price. This price is then broadcast to all DERs, who can allocate their production and consumption according to the price and their bid. The PowerMatcher concept was demonstrated in the PowerMatching City, consisting in 22 Dutch homes located in a suburb of Hoogkerk [26].

Another example of the price-based transactional control architecture is the Olympic Peninsula Project [20]. The testbed demonstration in the Olympic Peninsula, Washington, was designed, implemented and conducted by The Pacific Northwest National Lab [20]. The experiment was designed to deal with transmission and distribution congestion, load shifting and peak shaving. The set-up consisted of residential, commercial and municipal loads and distributed generators bidding into and responding to the local 5-minute market. The shadow market proposed in this project uses auction and biding process, which are settled a priori each every 5 minutes in order to establish a price. Appliances in the residential units offered voluntary response upon price signal. For commercial and municipal units, the price response was negotiated individually. A research on the market design for the price-based transactional control was done as a part of the ADDRESS project [27].

4) Autonomous control: Autonomously controlled power system services, for example frequency and voltage regulations have been a subject of research of many scientific papers. An autonomous frequency control in microgrid is presented in [28]. Asynchronous frequency and voltage control on a building level performed by separate devices was presented in [29].

\section{B. Internal operation}

The home automation system internal operation was divided into four categories: decision support scope, control strategy, fault handling and architecture.

1) Decision support system scope: An example of a system using knowledge representation and interpretation is an expert system using PROLOG for power system fault diagnosis [30]. The work presented in [30] is continued to propose Fault diagnosis using Petri net in [31]. A Predictive control example can be found in [32] and references within. A Predictive control system for a house heating system using a building thermal model is presented in [33]. This approach combined knowledge condensed in the building model, weather and power price prediction with user preferences to produce an optimal 24 hour building operation schedule. A HAS based on online adaptation algorithm is presented in [34], where the requests coming from the heating system are accepted or rejected on the basis of available power from the grid and internal rooms temperatures. The proposed scheduling algorithm is heuristic-driven, in favor of a best-effort solution. Therefore, the optimality of the on-line scheduling is not guaranteed. Such characteristic is traded-off with algorithm simplicity and ability to adapt to changing setup.

2) Control strategy: Many building controllers appearing in scientific papers are designed to target a single optimization 
problem and are not designed to change control strategy, for example [33] implementing a Model Predictive Control algorithm, and are called static in this control strategy classification. Other building controllers implementing flexible control strategy, usually called platforms, allows switching between different modes. For example device controller implemented in the Olympic Peninsula Project [20] is able to switch between two control strategies: on-line, externally controllable priceresponsive state to default, autonomous state, after the connection was lost. Both flexible and reconfigurable control strategy techniques were used in home automation system presented in [29]. In this system devices in a building were equipped in behavior descriptions - containers for different behavior patterns designed for a specific device type. A device that had many device description could switch from one strategy to another, for example when a connection was lost a heater switched from price based indirect control to autonomous frequency control. Machine learning techniques were used to modify its internal logic(policies) in the run-time. This HAS implemented very simple reconfiguration technique: code mobility, where policy for a device was designed externally, planted and run in the device, without restarting the software.

3) Fault handling: In the Olympic Peninsula Project [20] all controlled units in the building were equipped with local controllers that made decisions based on received price. In case of faults on the Internet communication link, lasting for more than 5 minutes, units switch to a default mode. The controller used both fault handling techniques first masking the failure attempted to reconnect for 5 minutes and then tolerating the failure - switched to the default control objective, user comfort, and was not responsive to the external signals.

4) Architecture: Centralized controller architecture was presented in [33]. A single module implements heating system optimization performing model predictive control with inputs of heaters state, weather conditions, weather prediction and a 24 hour prediction of power price, and output of the optimized schedule of power consumption minimizing the heating cont while maintaining user comfort. Hierarchical control architecture is presented in [35], where the Home Energy Manager (HEM) is controlling devices so as to meet user's comfort requirements and utility grid requests. The HEM has a layered structure, in which each layer is deputed to manage requests issued from devices, and it operates decisions basing on specific optimization objectives and resource allocation. Each device communicates its consumption request to the HEM, which computes a priority-based execution pool using information on users comfort settings, processes deadlines and operational constraints. The lowest layer, so-called Admission Controller, manages the requests in real time with an online scheduling logic. Requests that are rejected, because of lack of available power or low priority, are bounced to the intermediate level, called Load Balancer, which is based on a mixedinteger optimization model. It schedules the requests in order to minimize the energy expenses, while respecting processes deadlines and capacity constraints. The third layer, called Demand Response Manager (DRM), is deputed to exchange information from the grid on load shaving, load shifting, and it can bid on energy market. Different MAS solutions for distributed HAS were presented in [36], [37], [38]. Agents are designed to represent different devices and coordinate their operation with other agents competing over available power from the shared grid connection.

\section{Building representation}

1) Single DER: Most of HAS for demand side management supervise all controllable units present in the building. When considering a building as a single DER, all global constrains (power, temperature, availability of devices) need to be managed locally by scheduling. For example HAS centrally optimize the device schedule based on some objectives: minimizing the energy price while maintaining user comfort [33] or maintain power consumption set-point while respecting device's operation time and power constrains [34]. With a single HAS or a gateway needed to interface to the external VPP controller, interoperability between all multi-vendor and multi-technology devices and the home gateway or controller becomes an issue. OGEMA (Open Gateway Energy Management Alliance) [39] offers a software platform providing methods for reading and sending data to heterogeneous devices in the house using specific, vendor defined drivers. OGEMA encompasses units description models and application drivers, and it is designed to be hardware-independent [39].

2) Collection of DERs: Distributed building control systems were proposed in many publications such as [37], [38], [40] with effective use of negotiation between subsystems or particular devices existing in the space. In this approach the devices' operation is scheduled to achieve overall system goal, for example energy conservation or maintaining aggregated power set-point. The flexibility interface [41], defining DER information model for communication between a VPP controller and the DER, was developed in iPower project. The flexibility interface scheme is proposed for every DER type. To represent a building in the flexibility interface information model, subsystems need to be represented as separate DERs. All house DERs, for example light ing and heating systems, need a separate flexibility interface model and are controlled independently. Interconnections and internal influences, for example between heating and ventilation subsystems, need to be addressed at the VPP level.

\section{CONTROL COMPARISON CRITERIA AND SIMULATION TOOLS}

In order to choose the right DER control strategy for a required Demand Side Management application, a comparison criteria need to be defined. In this paper we propose several requirements that can be a base of comparison between different control approaches. Examples of comparison criteria and a short description of how different control strategies fulfill specific requirement is presented in Table I.

The service delivery performance can be compared between different DER control approaches. This criteria depends on the offered service and optimization algorithm used to control DERs. The speed of activation and deactivation is needed for ancillary services, overall performance of DERs or its aggregation is important for load shedding service.

In order to quantify the comparison between different DER control approaches, a simulation tool or experimental environment is needed. Laboratory set-up might be to small to test scalability requirement and implement market based control strategies, large filed test might not be available for 
TABLE I. COMPARISON OF DER CONTROL STRATEGIES BASED ON CHOSEN REQUIREMENTS.

\begin{tabular}{|c|c|c|c|c|c|}
\hline \multicolumn{2}{|c|}{ Requirement } & \multicolumn{4}{|c|}{ DER control strategy } \\
\hline & & Autonomous control & Indirect control & Transactional control & Direct control \\
\hline \multirow[t]{5}{*}{ Communication } & $\begin{array}{l}\text { Communication } \\
\text { links }\end{array}$ & $\begin{array}{l}\text { Only for activation and deac- } \\
\text { tivation of the service. }\end{array}$ & $\begin{array}{l}\text { One way communication or an acces- } \\
\text { sible server with current signal. }\end{array}$ & Two way & Two way \\
\hline & $\begin{array}{l}\text { Size of exchanged } \\
\text { data }\end{array}$ & None & $\begin{array}{l}\text { Continuous signal: small packet; } \\
\text { schedule: defined size schedule. }\end{array}$ & $\begin{array}{l}\text { During negotiation time: small to } \\
\text { medium size of bids. }\end{array}$ & $\begin{array}{l}\text { During configuration time: detailed } \\
\text { information about the unit(large, } \\
\text { DER specific size); for continuous } \\
\text { signal: small packet, schedule: de- } \\
\text { fined size schedule. }\end{array}$ \\
\hline & $\begin{array}{l}\text { Frequency of data } \\
\text { exchange }\end{array}$ & None & $\begin{array}{l}\text { Continuous signal: } 5 \text { min-1 hour, } \\
\text { schedule: daily. }\end{array}$ & $\begin{array}{l}\text { Frequency of negotiations: hourly or } \\
\text { intra-hour. for every negotiation iter- } \\
\text { ation: frequent data exchange (intra- } \\
\text { second); rarely for service delivery } \\
\text { monitoring (intra-hour). }\end{array}$ & $\begin{array}{l}\text { For direct power control: depends } \\
\text { on the service offered, for exam- } \\
\text { ple congestion management } 1 \text { min; } \\
\text { scheduled operation: daily; delta con- } \\
\text { sumption and differed operation: for } \\
\text { example for load shedding is event } \\
\text { driven, could be sent daily. }\end{array}$ \\
\hline & $\begin{array}{l}\text { Communication in- } \\
\text { frastructure }\end{array}$ & $\begin{array}{l}\text { No communication needed, } \\
\text { no remote interfaces, acti- } \\
\text { vation deactivation interface } \\
\text { needed (can be manual). }\end{array}$ & $\begin{array}{l}\text { Wired (eg UDP over Ethernet, web } \\
\text { service), radio, GSM; no reliable con- } \\
\text { nection needed. }\end{array}$ & Reliable high-speed connection. & $\begin{array}{l}\text { Reliable connection, not necessarily } \\
\text { high-speed. }\end{array}$ \\
\hline & $\begin{array}{l}\text { Addressability of } \\
\text { DERs }\end{array}$ & None & Not required & Required & Required \\
\hline \multirow[t]{3}{*}{ Controllability } & $\begin{array}{l}\text { Dependency on } \\
\text { other parties }\end{array}$ & Independent & $\begin{array}{l}\text { Depended; if a schedule is provided } \\
\text { it can be independent for a period of } \\
\text { time. }\end{array}$ & Dependent & $\begin{array}{l}\text { Depended; with a schedule can be } \\
\text { independent for a period of time. }\end{array}$ \\
\hline & $\begin{array}{l}\text { Additional parties } \\
\text { (e.g. Aggreagator, } \\
\text { market) }\end{array}$ & No additional parties needed. & $\begin{array}{l}\text { Sophisticated external controller or } \\
\text { aggregator is needed with a prediction } \\
\text { of the DER behavior. For price-based } \\
\text { indirect control economical calcula- } \\
\text { tions of price are needed. }\end{array}$ & $\begin{array}{l}\text { Market infrastructure is needed. If } \\
\text { the minimum market bid is large, } \\
\text { aggregator is required. }\end{array}$ & $\begin{array}{l}\text { External controller with detailed in- } \\
\text { formation about the DER description, } \\
\text { capabilities (including flexibility) and } \\
\text { state. }\end{array}$ \\
\hline & $\begin{array}{l}\text { DER responsive- } \\
\text { ness to a control } \\
\text { signal }\end{array}$ & Not responsive & $\begin{array}{l}\text { The behavior of the DER is not cer- } \\
\text { tain, because of autonomous decisions } \\
\text { taken within DER. }\end{array}$ & $\begin{array}{l}\text { The DER response is certain, } \\
\text { guarded by market agreements and } \\
\text { monitored. }\end{array}$ & $\begin{array}{l}\text { The DER response is certain, stated } \\
\text { in the contract and monitored. }\end{array}$ \\
\hline \multirow[t]{2}{*}{ Markets } & Contract & No & No & $\begin{array}{l}\text { Yes, accepted marked bid is consid- } \\
\text { ered a short contract. }\end{array}$ & Yes, typically long term contract. \\
\hline & Market integration & No & Possible through external aggregator. & Yes & Possible through external aggregator. \\
\hline Security & $\begin{array}{l}\text { Privacy of DER } \\
\text { data exchange }\end{array}$ & $\begin{array}{l}\text { High privacy, no data ex- } \\
\text { change. }\end{array}$ & High privacy, no data exchange. & $\begin{array}{l}\text { Hight privacy since only bids are ex- } \\
\text { changed without information about } \\
\text { bidding strategy. }\end{array}$ & $\begin{array}{l}\text { Privacy issues can be a problem due } \\
\text { to exchange of detailed data about the } \\
\text { DER. }\end{array}$ \\
\hline Scalability & Scalable solution & Yes & Yes & Yes & No \\
\hline
\end{tabular}

such study. Simulation tools are very appropriate to produce quantitative results for comparison between different control approaches. A required simulation tool need to include domains of power system, and communication network, house and appliance models, HAS models. User behavior domain can also be added, especially in the context of control of residential houses. No such single simulation exist, but a co-simulation concept can be used to combine simulations from different domains. Ongoing international efforts in cosimulations in the context of the power system are resulting in creating frameworks to run several simulations together. The need to create multi-domain simulations was recognized European Energy Research Alliance (EERA) ${ }^{2}$, Joint Programme on Smart Grids published a list of useful simulations that can be combined [42]. An example of a promising framework for co-simulation is Mosaik ${ }^{3}$ developed by OFFIS. Mosaik a modular platform for the evaluation of Smart Grid control mechanisms and offers interfaces for many popular simulations [43], proposes a formal scenario description and a control specification [44]. Extension of Mosaik with a communication network is needed to simulate different DER control strategies and compare results on the same use case and scenario.

\section{CONCLUSION AND FUTURE WORK}

Power balance in grids with a high share of DER can be improved by buildings' responsiveness to signals or commands.

\footnotetext{
${ }^{2}$ http://www.eera-set.eu/

${ }^{3} \mathrm{http}: / /$ mosaik.offis.de/
}

Many demand response controllers have been implemented and tested, some were deployed and verified in field tests. Different approaches were studied in order to achieve effective, scalable and incorporable architecture solutions. This paper presented an overview on load control policies for demand side management in buildings, and proposes a framework DSM controllers classification based on different characteristics.

This classification can be used for comparing different load control policies for smart buildings, it provides an overview of different DSM systems, it can help in the definition of controller specifications, and put forward a point of view for controllers standardization. A quantitative comparison between different approaches is very important, but it can only be done when appropriate co-simulation tools are available in the near future.

\section{REFERENCES}

[1] A. European Commission, DG Energy, "Eu energy portal," 2011.

[2] A. Simon and R. Belles, "Estimated state level energy flows in 2008," Lawrence Livermore National Laboratory, Tech. Rep., 2009.

[3] “Aftale [...] om den danske energipolitik 2012-2020," Danish Energy Authority, Tech. Rep., March 2012.

[4] World Business Council for Sustainable Development (WBCSD), "Transforming the market: Energy efficiency in the buildings," 2009.

[5] M. Ifland, N. Exner, and D. Westermann, "Appliance of direct and indirect demand side management," in Energytech, 2011 IEEE, 2011, pp. 1-6. 
[6] K. Heussen, S. You, B. Biegel, L. H. Hansen, and K. B. Andersen, "Indirect control for demand side management - a conceptual introduction," in Innovative Smart Grid Technologies (ISGT Europe), 2012 3rd IEEE PES International Conference and Exhibition on, 2012, pp. 1-8.

[7] O. Gehrke and F. Isleifsson, "An aggregation friendly information model for demand side resources," in Local Computer Networks (LCN), 2010 IEEE 35th Conference on, oct. 2010, pp. 1019 -1023.

[8] N. C. Nordentoft, Y. Ding, L. H. Hansen, P. D. Cajar, P. Brath, H. W. Bindner, and C. Zhang, "Development of a dso-market on flexibility services," iPower, Tech. Rep., 2013.

[9] A. S. Tanenbaum and M. Van Steen, Distributed systems : principles and paradigms. Prentice Hall, 2007, vol. 2.

[10] K. Kok, G. Venekamp, and P. Macdougall, "Market-based control in decentralized electrical power systems," in First International Workshop on Agent Technologies for Energy Systems, ATES2010, Toronto, 2010.

[11] S. H. Clearwater, R. Costanza, M. Dixon, and B. Schroeder, "Saving energy using market-based control," Market Based Control. World Scientific: Singapore, pp. 253-273, 1996.

[12] R. S. Michalski, J. G. Carbonell, and T. M. Mitchell, Machine learning. An artificial intelligence approach. Morgan Kaufmann, 1986, vol. 2.

[13] C. G. Rieger, D. I. Gertman, and M. A. McQueen, "Resilient control systems: next generation design research," in Human System Interactions, 2009. HSI'09. 2nd Conference on. IEEE, 2009, pp. 632-636.

[14] R. Negenborn, B. De Schutter, and J. Hellendoorn, "Multi-agent model predictive control: A survey," 2004.

[15] A. Jones and A. Saleh, "A decentralized control architecture for computer integrated manufacturing systems," in Intelligent Control, 1989. Proceedings., IEEE International Symposium on, 1989, pp. 44-49.

[16] M. Sebastian, J. Marti, and P. Lang, "Evolution of dso control centre tool in order to maximize the value of aggregated distributed generation in smart grid," in SmartGrids for Distribution, 2008. IET-CIRED. CIRED Seminar, june 2008, pp. $1-4$.

[17] P. Vinter, "Introduction to power hub, confidential," DONG Energy, Tech. Rep., November 2011.

[18] D. E. A/S. Power hub - an important part of the smart grid.

[19] F. Schweppe, R. Tabors, J. Kirtley, H. Outhred, F. Pickel, and A. Cox, "Homeostatic utility control," Power Apparatus and Systems, IEEE Transactions on, vol. PAS-99, no. 3, pp. 1151 -1163, May 1980.

[20] D. J. Hammerstrom, P. Investigator, R. Ambrosio, T. A. Carlon, J. G. Desteese, R. Kajfasz, and R. G. Pratt, "Pacific Northwest GridWise Testbed Demonstration Projects Part I . Olympic Peninsula Project," Contract, p. 157, 2007. [Online]. Available: http://www.gridwise.pnl.gov/docs/op_project_final_report_pnnl17167.pdf

[21] F. L. and Alvarado, "Controlling power systems with price signals," Decision Support Systems, vol. 40, no. 34, pp. 495 - 504, 2005, challenges of restructuring the power industry. [Online]. Available: http://www.sciencedirect.com/science/article/pii/S0167923604001241

[22] P. Nørgaard, F. Sossan, and H. Nielsen, "Indirect regulation of many der units through broadcasted dynamic price signal," in 21st International Conference on Electricity Distribution, 2011.

[23] P. Pinson, T. Jonsson, H. Madsen, H. W. Bindner, H. Hansen, K. B. Andersen, L. H. Hansen, and G. C. Tarnowski, "Indirect control by prices basic concepts, applications and requirements," iPower, Tech. Rep., 2012. [Online]. Available: hhttp://www.ipower-net.dk/ /media/iPower/Documents/iPowerFlexInterface-IndirectControl.ashx

[24] F. Sossan and H. Bindner, "Evaluation of the performance of indirect control of many dsrs using hardware-in-the-loop simulations," in Decision and Control (CDC), 2012 IEEE 51st Annual Conference on. IEEE, 2012, pp. 5586-5591.

[25] F. Bliek, A. van den Noort, B. Roossien, R. Kamphuis, J. de Wit, J. van der Velde, and M. Eijgelaar, "Powermatching city, a living lab smart grid demonstration," in Innovative Smart Grid Technologies Conference Europe (ISGT Europe), 2010 IEEE PES, oct. 2010, pp. 1 -8 .

[26] F. Bliek, A. van den Noort, B. Roossien, R. Kamphuis, J. de Wit, J. van Der Velde, and M. Eijgelaar, "Powermatching city, a living lab smart grid demonstration," in Innovative Smart Grid Technologies Conference Europe (ISGT Europe), 2010 IEEE PES. IEEE, 2010, pp. 1-8.
[27] T. Kostic, C. Effantin, and E. Lambert, "How to increase interoperability in european smartgrid projects? the address experience regarding model driven integration based on international standards," 2012, pp. 652-657.

[28] P. Piagi and R. H. Lasseter, "Autonomous control of microgrids," in Power Engineering Society General Meeting, 2006. IEEE. IEEE, 2006, pp. 8-pp.

[29] D. Kullmann, O. Gehrke, and H. Bindner, "Asynchronous control of distributed energy resources using behaviour descriptions," in Computing, Networking and Communications (ICNC), 2012 International Conference on. IEEE, 2012, pp. 216-220.

[30] S. Jadid, B. Jeyasurya, and S. Khaparde, "Power system fault diagnosis expert system using prolog," in TENCON '89. Fourth IEEE Region 10 International Conference, 1989, pp. 778-781.

[31] Z. Tao, C. Qing, and G. Zhanjun, "Fault diagnosis of 3/2 breaker scheme in substation based on petri net," in Electric Utility Deregulation and Restructuring and Power Technologies (DRPT), 2011 4th International Conference on, 2011, pp. 348-352.

[32] I. Lampropoulos, P. P. van den Bosch, and W. L. Kling, "A predictive control scheme for automated demand response mechanisms," in Innovative Smart Grid Technologies (ISGT Europe), 2012 3rd IEEE PES International Conference and Exhibition on. IEEE, 2012, pp. 1-8.

[33] Y. Zong, D. Kullmann, A. Thavlov, O. Gehrke, and H. Bindner, "Model predictive control strategy for a load management research facility in the distributed power system with high wind penetration-towards a danish power system with 50\% wind penetration," in Power and Energy Engineering Conference (APPEEC), 2011 Asia-Pacific. IEEE, 2011, pp. $1-4$.

[34] G. Costanzo, A. Kosek, G. Zhu, L. Ferrarini, M. Anjos, and G. Savard, "An experimental study on load-peak shaving in smart homes by means of online admission control," in Innovative Smart Grid Technologies (ISGT Europe), 2012 3rd IEEE PES International Conference and Exhibition on, 2012, pp. 1-8.

[35] G. Costanzo, G. Zhu, M. Anjos, and G. Savard, "A system architecture for autonomous demand side load management in smart buildings," Smart Grid, IEEE Transactions on, vol. 3, no. 4, pp. 2157-2165, 2012.

[36] Z. Wang, R. Yang, and L. Wang, "Multi-agent control system with intelligent optimization for smart and energy-efficient buildings," in IECON 2010-36th Annual Conference on IEEE Industrial Electronics Society. IEEE, 2010, pp. 1144-1149.

[37] A. M. Kosek and O. Gehrke, "A distributed multi-agent control system for power consumption in buildings," in Communicating Process Architectures 2012, 2012.

[38] S. Abras, S. Ploix, S. Pesty, and M. Jacomino, "A multi-agent design for a home automation system dedicated to power management," in $A r$ tificial Intelligence and Innovations 2007: from Theory to Applications. Springer, 2007, pp. 233-241.

[39] H. W. David Nestle, Jan Ringelstain, "Open energy gateway architecture for customers in the distribution grid," Fraunhofer IWES, Tech. Rep., December 2009.

[40] P. Davidsson and M. Boman, "A multi-agent system for controlling intelligent buildings," in MultiAgent Systems, 2000. Proceedings. Fourth International Conference on. IEEE, 2000, pp. 377-378.

[41] B. Biegel, C. H. Lyhne, D. V. Tackie, K. B. Andersen, L. H Hansen, M. A. Kjaer, P. Andersen, and T. Green, "Flexibility interface information modeling for direct control," iPower project, Tech. Rep., 2011. [Online]. Available: http://www.ipowernet.dk/ /media/iPower/Documents/fi.ashx

[42] H. Bindner and M. Marinelli, "Overview of Simulation Tools for Smart Grid," EERA JP on Smart Grid SubProgramme 2 Energy Management, Tech. Rep., May 2013.

[43] S. Schütte, S. Scherfke, and M. Sonnenschein, "mosaik-smart grid simulation api," in Proceedings of SMARTGREENS 2012-International Conference on Smart Grids and Green IT Systems, 2012, pp. 14-24.

[44] S. Schütte, "Composition of simulations for the analysis of smart grid scenarios," in Energieinformatik, 2011. 